\title{
ACTITUDES HACIA LA INVESTIGACIÓN DE INTERNOS DE OBSTETRICIA ROTANTES EN UN HOSPITAL DE LIMA-PERÚ
}

\author{
ATTITUDES TOWARDS THE INVESTIGATION OF ROTATING OBSTETRICS INMATES IN A LIMA-PERU HOSPITAL
}

\author{
John Barja-Ore', Óscar Otoya-Petit², Emilio Oswaldo Vega-Gonzales³, \\ Nelly Moreno-Gutierrez4, Rudi Amalia Loli Ponce ${ }^{1}$
}

\begin{abstract}
RESUMEN
Objetivo: Determinar las actitudes hacia la investigación de internos de obstetricia rotantes en el Hospital Sergio E. Bernales (HSEB) durante diciembre 2018 a enero 2019. Métodos: Estudio transversal. La población estuvo constituida por internos de obstetricia que realizaban prácticas profesionales a quienes se les aplicó una escala validada de 28 ítems distribuidos en 6 dimensiones, que evaluó actitudes hacia la investigación. Resultados: Participaron del estudio 57 internos de obstetricia. La edad promedio fue de $23,5 \pm 2,1$ años y $96,5 \%$ eran de sexo femenino. El $54,4 \%$ de internos presentó actitud regular hacia la investigación científica, el $24,6 \%$ tuvo una buena actitud y $21 \%$ una mala actitud. El $93,0 \%$ de internos estuvo de acuerdo o muy de acuerdo con que la publicación en una revista ayuda a mejorar la práctica clínica, la mayoría se sentía capaz de interpretar un artículo científico $(68,4 \%)$ y preparados para realizar una investigación relacionada con la carrera (54,3\%); por otro lado, consideraban que para realizar investigaciones de calidad en la universidad debían contar con herramientas básicas físicas y virtuales $(94,7 \%)$ y que el curso de redacción científica debía centrarse en la enseñanza de la elaboración de un artículo (84,2\%). La mayoría estuvo muy en desacuerdo y en desacuerdo en optar por otra modalidad de graduación que no sea la tesis (50,9\%). Conclusión: Más de la mitad de los internos de obstetricia del HSEB tenían actitud regular hacia la investigación. Los resultados evidencian la necesidad de continuar fortaleciendo y promoviendo la investigación en los estudiantes de obstetricia.
\end{abstract}

Palabras clave: Actitud; Investigación; Estudiantes; Obstetricia. (fuente: DeCS BIREME)

\begin{abstract}
Objective: Determine attitudes towards the investigation of rotating obstetrics inmates at Sergio E. Bernales Hospital (HSEB) during December 2018 to January 2019. Methods: Transversal study. The population was made up of obstetrics interns who performed professional practices to whom a validated scale of 28 items distributed in 6 dimensions was applied, which assessed attitudes towards research. Results: 57 obstetrics interns participated in the study. The average age was $23.5 \pm 2.1$ years and $96.5 \%$ were female. $54.4 \%$ of inmates presented a regular attitude towards scientific research, $24.6 \%$ had a good attitude and $21 \%$ had a bad attitude. $93.0 \%$ of inmates agreed or strongly agreed that publication in a journal helps improve clinical practice, most felt able to interpret a scientific article (68.4\%) and prepared to conduct research career related (54.3\%); On the other hand, they considered that in order to carry out quality research in the university they had to have basic physical and virtual tools $(94.7 \%)$ and that the scientific writing course should focus on teaching the preparation of an article (84.2\%). The majority disagreed and disagreed in opting for another graduation modality other than the thesis (50.9\%). Conclusion: More than half of HSEB's obstetric inmates had a regular attitude toward research. The results show the need to continue strengthening and promoting research in obstetrics students.
\end{abstract}

Key words: Attitude; Research; Students; Obstetrics. (source: MeSH NLM)

${ }^{1}$ Facultad de medicina. Universidad Nacional Mayor de San Marcos, Lima-Perú

${ }^{2}$ Departamento de Gineco-Obstetricia. Hospital Sergio E. Bernales, Lima-Perú.

${ }^{3}$ Universidad Cesar Vallejo Sede Lima Este, Lima-Perú.

${ }^{4}$ Escuela Profesional de Obstetricia. Universidad de San Martín de Porres, Lima-Perú.

Citar como: John Barja-Ore, Óscar Otoya-Petit, Emilio Oswaldo Vega-Gonzales, Nelly Moreno-Gutierrez, Rudi Amalia Loli Ponce. Actitudes hacia la investigación de internos de obstetricia rotantes en un hospital de Lima-Perú. Rev. Fac. Med. Hum. Octubre 2019; 19(4):53-59. DOI 10.25176/RFMH.v19i4.2341

Artículo publicado por la Revista de la Facultad de Medicina Humana de la Universidad Ricardo Palma. Es un artículo de acceso abierto, distribuído bajo los términos de la Licencia Creative Commons: Creative Commons Attribution 4.0 International, CC BY 4.0 (https://creativecommons.org/licenses/by/4.0/), que permite el uso no comercial, distribución y reproducción en cualquier medio, siempre que la obra original sea debidamente citada. Para uso comercial, por favor póngase en contacto con revista.medicina@urp.pe 


\section{INTRODUCCIÓN}

La universidad es una institución que constituye el nexo directo entre la sociedad y la ciencia, dado que contribuye a la resolución de los problemas que se generan en diversas áreas (como la salud), a partir del uso de los nuevos conocimientos producidos a través de la investigación, reconociendo a esta como una de sus funciones primordiales', para que esta función se ejecute adecuadamente, es necesario que las facultades o escuelas de las universidades no sólo reconozcan la trascendencia de las competencias investigativas que deben adquirir los estudiantes de pregrado, sino que contribuyan a desarrollarlas $y$ afianzarlas como hábitos profesionales².

La investigación es uno de los ejes transversales de la malla curricular y del proceso enseñanza aprendizaje durante la formación profesional; no obstante, esto no siempre se consigue puesto que muchos estudiantes no le otorgan la debida importancia, desconociéndola como una herramienta útil para su desarrollo ${ }^{3}$, pese a esta realidad, es necesario que todo estudiante aprenda cómo realizar investigación a fin de generar nuevo conocimiento 4 .

La formación en investigación y el desarrollo de esta en los estudiantes de ciencias de la salud de pregrado es esencial debido a que en la labor diaria de un profesional se recoge información, se analiza, se plantean posibles respuestas, se establece un diagnóstico presuntivo y se realiza seguimiento a los pacientes con el fin de comprobar lo inicialmente planteado ${ }^{5}$, asimismo, la investigación permite orientar un perfil profesional hacia la resolución de determinadas situaciones que tienen un gran impacto en la salud pública de un país, en este sentido, se requiere que exista una predisposición a fomentarla y desarrollarla en los propios estudiantes ${ }^{6}$.

Las actitudes hacia la investigación se encuentran íntimamente vinculadas con las capacidades y competencias de los docentes y la de los estudiantes las cuales dependerán esencialmente del contexto educativo en el que se desarrollen. El estudio de estas actitudes refleja un enfoque crítico de la formación y permite una oportunidad para implementar medidas de calidad en el ámbito universitario7.

En el Perú, según la Superintendencia Nacional de Educación Superior Universitaria (SUNEDU), el número de documentos citables es uno de los indicadores para medir la actividad de investigación universitaria; al respecto, se ha evidenciado un incremento de dichos documentos, de 927 en el año 2010 a 1610 para el año 2015, siendo esta información especialmente relevante para comprender el interés de la investigación por parte de los estudiantes universitarios a nivel nacional ${ }^{8}$.

La formación profesional del estudiante de obstetricia involucra mucho más que el aprendizaje de la atención clínica del embarazo, parto o puerperio, o del abordaje de la salud sexual y reproductiva de la mujer en edad fértil, sino también el desarrollo de competencias en investigación. Por tal motivo, el presente estudio se desarrolló con el objetivo de determinar las actitudes hacia la investigación que tienen los internos de obstetricia rotantes en el Hospital Sergio E. Bernales durante diciembre 2018 a enero 2019.

\section{MÉTODOS}

Estudio transversal. La población estuvo constituida por internos de obstetricia de la Universidad Nacional Mayor de San Marcos (UNMSM), Universidad Nacional Federico Villarreal (UNFV), Universidad de San Martín de Porres (USMP), Universidad Arzobispo Loayza (UAL) y Universidad Alas Peruanas (UAP) los cuales se encontraban rotando en el Hospital Sergio Bernales. Los internos fueron seleccionados de acuerdo a criterios de inclusión y exclusión:

- Criterios de inclusión: Internos de obstetricia de universidades nacionales o privadas que hayan realizado al menos una rotación del internado en el Hospital Sergio Bernales.

- Criterios de exclusión: No aceptar participar voluntariamente de la investigación o encontrarse rotando en otra sede. Se aplicó a los internos una escala de evaluación de actitudes hacia la investigación para estudiantes universitarios, la cual cuenta con 27 ítems y cinco categorías de respuesta establecidas mediante escala de Likert. La escala ha sido validada (validez de contenido y constructo) en Perú por Arellana-Sacramento ${ }^{9}$ quien obtuvo un coeficiente de alfa de Cronbach de 0,65 para dicho instrumento. La escala de actitudes está conformada por seis dimensiones:

» Actitud hacia la elaboración de artículos científicos

» Participación en investigación

» Acciones de la universidad para incentivar la investigación en el alumno

» Parámetros en la investigación dentro de la universidad

» Posibilidad de realizar investigación dentro de la universidad

» Revisión de material de investigación. 
El valor final de las actitudes se determinó según el Baremo del instrumento: por medio de la suma de puntajes de los reactivos, siendo una actitud "buena" cuando el puntaje fue mayor o igual de 103; actitud "regular" cuando el puntaje estuvo entre 93 y 102; y actitud "mala" cuando el puntaje fue menor o igual de 92 puntos.

El análisis estadístico fue realizado con el programa SPSS versión 25 para Windows. Se empleó estadística univariada basada en la obtención de frecuencias, porcentajes, medidas de tendencia central y de dispersión.

Con relación a los aspectos éticos, la participación de los internos de obstetricia fue voluntaria y confidencial para lo cual firmaron un consentimiento informado. El proyecto fue aprobado para su ejecución por la Oficina de Docencia e Investigación del Hospital Sergio Bernales.

\section{RESULTADOS}

La población estuvo constituida por 69 internos de obstetricia; sin embargo, 12 internos, no desearon participar en la investigación quedando disponibles 57 para el análisis. La edad promedio fue de 23,5 $\pm 2,1$ años, el 71,9\% de los internos tuvieron entre 20 y 24 años y $96,5 \%$ eran de sexo femenino. El 36,6\% de los internos fueron de la UNMSM, 28,1\% de la UAL y $7 \%$ de la USMP (Tabla 1).

Tabla 1. Características de los internos de obstetricia.

\begin{tabular}{llll}
\multicolumn{1}{c}{ Características de los internos } & & $\mathbf{N}^{\circ}$ & $\%$ \\
Edad & & & \\
20 a 24 años & 41 & 28,1 \\
25 a 33 años & 16 & \\
Sexo & & 96,5 \\
Femenino & 55 & 3,5 \\
Masculino & 2 & \\
Universidad & & 36,6 \\
UNMSM & 22 & 28,1 \\
UAL & 16 & 14,0 \\
UNFV & 8 & 12,3 \\
UAP & 7 & 7,0 \\
USMP & 4 & 100 \\
Total & 57 &
\end{tabular}

La mayor frecuencia de internos presentó una actitud regular hacia la investigación científica (54,4\%); mientras que, el $24,6 \%$ tuvo una buena actitud y (Tabla 2).

Tabla 2. Actitud hacia la investigación científica según universidades.

\begin{tabular}{|c|c|c|c|c|c|c|}
\hline \multirow{3}{*}{ Universidad } & \multicolumn{6}{|c|}{ Actitud hacia la investigación } \\
\hline & \multicolumn{2}{|c|}{ Mala } & \multicolumn{2}{|c|}{ Regular } & \multicolumn{2}{|c|}{ Buena } \\
\hline & $\mathbf{N}^{\circ}$ & $\%$ & $\mathbf{N}^{\circ}$ & $\%$ & $\mathbf{N}^{\circ}$ & $\%$ \\
\hline UNMSM & 6 & 27,3 & 9 & 40,9 & 7 & 31,8 \\
\hline UAL & 3 & 18,8 & 9 & 56,2 & 4 & 25,0 \\
\hline UNFV & 1 & 12,5 & 7 & 87,5 & 0 & 00 \\
\hline UAP & 2 & 28,6 & 3 & 42,8 & 2 & 28,6 \\
\hline USMP & 0 & 0 & 3 & 75 & 1 & 25,0 \\
\hline
\end{tabular}


Con relación a las actitudes hacia la elaboración de artículos científicos se observó que la mayor frecuencia de internos estuvo de acuerdo o muy de acuerdo con que la publicación en una revista ayuda a mejorar la práctica clínica (93,0\%), la investigación sólo es importante cuando se realiza una maestría, doctorado o especialidad $(66,7 \%)$ y que los docentes universitarios deberían realizar investigaciones en la universidad (93,0\%). Respecto de las actitudes hacia la participación en investigación, la mayoría se sentía capaz de interpretar un artículo científico (68,4\%) y preparados para realizar una investigación relacionada con la carrera $(54,3 \%)$. En la dimensión relacionada a las acciones de la universidad para incentivar la investigación en el alumno, la mayoría de los internos consideraba que si pedían asesoría a un docente este podía aprovecharse de su trabajo $(59,6 \%)$ y que para realizar investigaciones de calidad en la universidad debían contar con herramientas básicas físicas y virtuales $(94,7 \%)$. (Tabla 3 )

Tabla 3. Actitudes hacia las dimensiones de elaboración de artículos científicos, participación en investigación y acciones de la universidad para incentivar la investigación en el alumno.

\section{Dimensiones}

\section{MD}

DA MDA

Actitudes hacia la elaboración de artículos científicos

La publicación en una revista científica ayuda a mejorar la práctica clínica

La investigación sólo es importante cuando realizas una maestría, doctorado o especialidad

Los docentes universitarios deberían realizar investigaciones en la universidad

Los cursos de investigación deberían complementarse fuera de horarios de clase

La investigación refuerza la formación académica universitaria

Asistir por decisión propia a eventos científicos y académicos

$\begin{array}{ccccc}0 & 0 & 5,3 & 52,6 & 42,1 \\ 5,3 & 10,4 & 21,1 & 42,1 & 21,1\end{array}$

NA/ND

DA

Actitudes hacia la participación en investigación

Me siento capaz de juzgar e interpretar un artículo científico de mi carrera

$\begin{array}{ccrrr}0 & 7 & 26,6 & 52,6 & 15,8 \\ 3,5 & 19,2 & 24,6 & 31,6 & 21,1 \\ 5,3 & 14 & 24,6 & 45,6 & 10,5 \\ 1,8 & 10,6 & 33,3 & 36,8 & 17,5\end{array}$

Conozco todos los requisitos para realizar una tesis en $\mathrm{mi}$ universidad

0

3,5

21,1

40,3

35,1

0

He consultado por mi cuenta al menos una revista científica este mes

Me siento preparado para realizar una investigación relacionada con mi carrera

40,4

26,3

49,1

Actitudes hacia las acciones de la universidad para incentivar la investigación en el alumno

Considero que los docentes no tienen tiempo para investigar

$8,8 \quad 17,5 \quad 36,8 \quad 28,1 \quad 8,8$

Si pido asesoría a un docente éste va a aprovecharse de mi trabajo

$5,3 \quad 5,3 \quad 29,8 \quad 43,9 \quad 15,7$

La universidad no nos incentiva a que investiguemos

$5,3 \quad 24,6 \quad 21 \quad 35,1 \quad 14$

Para realizar investigaciones de calidad en la universidad se debe contar con herramientas básicas físicas y virtuales

$\begin{array}{lllll}0 & 0 & 5,3 & 45,6 & 49,1\end{array}$

La investigación científica es la primera actividad que debe realizarse en la universidad

$0 \quad 0 \quad 21,1 \quad 47,3 \quad 31,6$

MD: Muy en desacuerdo; ED: En desacuerdo; NA/ND: Ni de acuerdo ni en desacuerdo; DA: De acuerdo; MDA: Muy de acuerdo. 
Con respecto a las actitudes hacia los parámetros en la investigación en la universidad se evidenció que el mayor porcentaje de internos estuvo de acuerdo o muy de acuerdo con que el curso de redacción científica debe centrarse en la enseñanza de la elaboración de un artículo (84,2\%) y en la participación en un grupo de investigación $(80,7 \%)$. Referente a las actitudes hacia la posibilidad de realizar una investigación dentro de la universidad, la mayoría estuvo muy en desacuerdo y en desacuerdo en optar por otra modalidad de graduación que no sea la tesis $(50,9 \%)$, no obstante, la mayoría estuvo muy de acuerdo y de acuerdo en que el docente debe ser un modelo a seguir en lo que se refiere a la investigación $(87,7 \%)$. En cuanto a las actitudes hacia la revisión de material bibliográfico, la mayoría estuvo de acuerdo o muy de acuerdo en que el estudiante que realiza publicaciones científicas tiene ventaja en el quehacer profesional y que un buen profesional es quien ha desarrollado investigaciones científicas durante la universidad.

Tabla 4. Actitudes en las dimensiones de parámetros en la investigación dentro de la universidad, posibilidad de realizar una investigación dentro de la universidad y revisión de material de investigación.

\section{Dimensiones}

MD

ED NA/ND

DA

MDA

Actitudes hacia los parámetros en la investigación dentro de la universidad

El que una investigación sea publicada en una revista científica garantiza la rigurosidad del proceso metodológico de investigación

El curso de redacción debe enfocarse en enseñar a elaborar un artículo científico
12,

(1)

0

7
43,8

0

15,8

49,1

35,1

Participar en grupos de investigación conformados por alumnos de la universidad

0

Los deseos de investigar desaparecieron mientras avanzaban los ciclos

$28,1 \quad 33,3 \quad 22,8 \quad 8,8$

Actitudes hacia la posibilidad de realizar investigación dentro de la universidad

Optar por otra modalidad de graduación en vez de realizar una tesis

$\begin{array}{ccccc}22,8 & 28,1 & 31,6 & 12,2 & 5,3 \\ 0 & 0 & 12,3 & 45,6 & 42,1 \\ 0 & 15,8 & 15,8 & 47,3 & 21,1 \\ 5,3 & 0 & 31,5 & 38,6 & 24,6 \\ 15,8 & 22,8 & 42,1 & 17,5 & 1,8\end{array}$

El docente universitario debe ser el modelo a seguir sobre investigación dentro de la universidad

No saber inglés es una limitación para realizar una buena investigación

Una persona inteligente tiene más ventaja para realizar una investigación

Los cursos ocupan mucho tiempo e impiden realizar una investigación

Actitudes hacia la revisión de material de investigación

El estudiante que publica revistas científicas tiene ventaja en el buen ejercicio de la carrera

$\begin{array}{crrrr}0 & 8,8 & 22,8 & 40,4 & 28,1 \\ 12,3 & 31,6 & 31,6 & 19,3 & 5,2 \\ 1,8 & 15,8 & 33,3 & 31,6 & 17,5\end{array}$

El resumen de los artículos científicos permite reconocer la fiabilidad de la investigación

Un buen profesional es aquel que ha investigado en la universidad

MD: Muy en desacuerdo; ED: En desacuerdo; NA/ND: Ni de acuerdo ni en desacuerdo; DA: De acuerdo; MDA: Muy de acuerdo. 


\section{DISCUSIÓN}

La presente investigación muestra que la mayoría de los internos de obstetricia tiene actitudes regulares hacia la investigación, esto es similar a lo hallado en un estudio realizado en Lima, el cual reporta que el $81,7 \%$ de los estudiantes de obstetricia presentaron actitudes de indiferencia ${ }^{10}$, tales resultados, resaltan la necesidad reforzar la investigación formativa desde los primeros años de estudio y de manera transversal en todas las asignatura profesionales, a través de talleres o programas educativos curriculares y extracurriculares orientados al perfeccionamiento de la investigación ${ }^{11}$; en este contexto, algunos autores demostraron que el interés hacia la investigación, así como las habilidades vacacional, concluyendo que el programa, así como otras estrategias, pueden tener un impacto positivo sobre el perfil de los estudiantes y egresados ${ }^{12}$. En un estudio realizado en internos de enfermería de la UNMSM $^{13}$, el $47 \%$ presentó una actitud favorable hacia la investigación.

En el análisis de las dimensiones de la variable de estudio, se resaltan aspectos como una tendencia positiva a considerar que la producción científica de docentes y estudiantes publicada en revistas de impacto contribuye a la mejora de la praxis clínica de los profesionales de la salud, en tal sentido, promover la investigación en los estudiantes universitarios se perfila como un eje crítico para el desarrollo, lo cual se ve reforzado por los hallazgos de un estudio realizado en Honduras que demostró que 9 de cada 10 estudiantes de medicina refirieron que la investigación científica aporta significativamente en la producción científica de un país ${ }^{14}$.

En el análisis de actitudes hacia las acciones de la universidad para incentivar la investigación, una parte importante de internos de obstetricia consideró que la universidad no incentiva la investigación; no obstante, la UNMSM cuenta un Programa de promoción de tesis de pregrado, en cual financia, previo concurso, las investigaciones de los estudiantes de las 5 escuelas profesionales que conforman la Facultad de Medicina. Los internos también consideraron que algunos docentes podían aprovecharse del estudio, situación que debe ser convenida desde la asesoría de la tesis, ya que los asesores pueden ser considerados coautores cuando los resultados de la investigación pretenden difundirse en artículos de investigación. Las universidades deben tener políticas de investigación que contemplen múltiples estrategias y recursos para fomentar y desarrollar la investigación formativa y la investigación científica, así en muchas universidades la tutoría o asesoría de investigación se perfila como una de los más relevantes, dado que se ha demostrado que su rol en la enseñanza de la metodología de investigación permite un aprendizaje significativo de este proceso ${ }^{15}$, Al respecto, un estudio realizado en estudiantes de Odontología de una universidad de Lima, muestra una realidad que refuerza esta idea, ya que se reportó que la asesoría inadecuada fue la principal limitación para realizar investigaciones ${ }^{16}$.

Referente a la actitud hacia la participación en investigación y la posibilidad de realizarla dentro de la universidad, se aprecia una inclinación positiva a estar preparado para realizar una investigación, así como el deseo y disposición de titularse bajo la modalidad de tesis y no por examen. En este marco, la información contrasta con un estudio donde una baja proporción de estudiantes de medicina presentaron una actitud adecuada hacia la investigación; no obstante, no se encontraban participando en la ejecución de algún proyecto ${ }^{17}$; así mismo, en otra investigación en estudiantes de enfermería, se reportó que $80,6 \%$ no habían participado de algún proyecto de investigación en lo que va de su formación profesional ${ }^{18}$. Con estos datos queda en evidencia que la predisposición de realizar investigaciones debe concretizarse durante y al término de la etapa universitaria, para lo cual resulta fundamental una mejora de la gestión universitaria, y en consecuencia, potenciar significativamente las capacidades investigativas de los estudiantes, a través de una educación continua e integradora ${ }^{19}$.

En la dimensión sobre la revisión de material de investigación, se evidencia que aproximadamente 3 de cada 10 internos estaban de acuerdo en que el resumen de un artículo de investigación no permite determinar su fiabilidad; además, la mayoría estaba de acuerdo en que si el estudiante publica tiene ventajas en el ejercicio de su carrera. Si bien esta información es favorable, se necesita que las universidades, a través de los gestores y formadores educativos, fomenten la capacitación permanente en el uso de las herramientas tecnológicas como los gestores bibliográficos y las bases de datos, búsqueda sistemática de las fuentes de información bibliográfica, así como el análisis crítico de estas, para su correcta interpretación, a fin de promover la producción de nuevos conocimientos con un adecuado respaldo teórico, metodológico y estadístico.

Entre las limitaciones del estudio se tiene que los internos de obstetricia seleccionados fueron sólo de un hospital; sin embargo, es posible que estas 
condiciones sean similares en internos de otros hospitales por lo que los resultados de este estudio podrían ser aplicables; a pesar de ello, resulta necesario realizar una investigación multicéntrica con una muestra representativa, Otra limitación fue la escasa documentación de estudios realizados con estudiantes de la carrera profesional de obstetricia.

\section{CONCLUSÓN}

En conclusión, más de la mitad de los internos de obstetricia del Hospital Sergio E. Bernales tenían actitud regular hacia la investigación, Los resultados evidencian la necesidad de continuar fortaleciendo y promoviendo la investigación como eje transversal en la formación de los estudiantes de la carrera de obstetricia.
Contribuciones de autoría: Los autores participaron en la génesis de la idea, diseño de proyecto, recolección e interpretación de datos, análisis de resultados y preparación del manuscrito del presente trabajo de investigación.

Financiamiento: Autofinanciado.

Conflicto de interés: Los autores declaran no tener conflicto de interés en la publicación de este artículo.

Recibido: 20 de marzo del 2019

Aprobado: 29 de agosto del 2019

Correspondencia: John Barja-Oré

Dirección: Prolongación Húsares de Junín N²01. Urbanización El Retablo, Comas. Teléfono: 951792628

Correo:johnbo247@gmail.com

\section{REFERENCIAS BIBLIOGRÁFICAS}

1. Sancén F. La universidad del futuro. Reencuentro. Análisis de los problemas universitarios. 2013;(68):10-8.

2. Sánchez-Carlessi H. La investigación formativa en la actividad curricular Rev Fac Med. Hum. 2017;17(2):71-4.

3. Peña C. La Importancia de la Investigación en la Universidad: Una Reivindicación del Sapere Aude Kantiano. Revista Amauta. 2015;25:79-85.

4. Rosario F, Chamorro Y, Moreno R. Actitudes hacia la investigación y rendimiento académico en estudiantes de una universidad privada de Lima. Psiquemag. 2016;5(1):255-75.

5. Carrizo J. Importancia de la investigación en la formación de Pregrado. Panorama Cuba y Salud. 2010;5(3):3-4.

6. Rodríguez T, Rodríguez A, García M. La investigación y su contribución formativa en estudiantes de las ciencias médicas. Edumecentro. 2016;8(1):143-58

7. Rojas $M$, Méndez R. Procesos de formación en investigación en la Universidad: ¿Qué le queda a los estudiantes? Sophia. 2017;13(2):53-69.

8. Superintendencia Nacional de Educación Superior Universitaria Informe bienal sobre la realidad universitaria peruana. $1^{\text {a }}$ edición. LimaPerú: Arte Perú S.A.C.; 2017. pp.67.

9. Arellano-Sacramento C, Hermoza-Moquillaza R, Elías-Podestá M, Ramírez-Julca M. Actitud hacia la investigación de estudiantes universitarios en Lima, Perú. FEM (Ed. impresa). 2017;20(4):191-97.

10. Yace J, Guerrero J, Delgado J. Nivel de actitud hacia la investigación científica y la estadística en estudiantes de obstetricia pregrado, Universidad Norbert Wiener, Agosto 2017. En: VIII Simposio Jornada de Investigación 2017. Lima: Perú; 2017. p. 37-39.

11. Barbón O, Bascó E. Clasificación de la actividad científica estudiantil en la educación médica superior. Educ Med. 2016;17(2):55-60.
12. Sánchez-Duque J, Rueda-Lizarazo L, Tafur-Puentes D, López-Serna M, Muñoz-HernandezD, Bados-EnriquezD, et al. Programa de entrenamiento vacacional en investigación: una propuesta de estudiantes de medicina colombianos. Educ Med. 2018;19(5):258-63.

13. Quispe Carmelo, M. Actitudes de los internos de enfermería hacia la investigación y la elaboración de tesis para titularse 2014 [Tesis de Licenciatura]. Lima-Perú: Universidad Nacional Mayor de San Marcos; 2015.

14. Ramírez A, Díaz-Valle D, Rivas K, Yanez V, Valle-Reconco J. Conocimientos, actitudes y prácticas en investigación de estudiantes de la carrera de medicina, Tegucigalpa - UNAH. Rev Fac Cienc Méd. 2016;13(1):10-17.

15. Amor M, Serrano R. El asesor académico: un nuevo perfil para la orientación y la tutoría en la universidad. Orientac Soc. 2016;16:15-23.

16. Castro Y, Sihuay-Torres K, Perez-Jiménez V. Producción científica y percepción de la investigación por estudiantes de odontología. Educ Med. 2018;19(1):19-22.

17. Cabrera-Enríquez JA, Cruzado-Mendoza C, Purizaca-Rosillo N, LópezSamanamú RO, Lajo-Aurazo Y, Peña-Sánchez ER, et al. Factores asociados con el nivel de conocimientos y la actitud hacia la investigación en estudiantes de medicina en Perú, 2011. Rev Panam Salud Publica. 2013;33(3):166-73.

18. Fajardo-Ramos E, Henao-Castaño ÁM, Vergara-Escobar OJ. La investigación formativa, perspectiva desde los estudiantes de enfermería. Salud Uninorte. 2015;31(3):558-64.

19. Hernández $E$, Losada J. A propósito de la investigación científica estudiantil en la educación médica ecuatoriana. Educ Med. 2018;19(6):319-80. 